\title{
Designing Foundations with Piles for Vibrating Machinery
}

\author{
M. Gohnert ${ }^{*}$, I. Luker $^{1}$ and C. Morris ${ }^{2}$ \\ ${ }^{I}$ School of Civil and Environmental Engineering, University of the Witwatersrand, South Africa; ${ }^{2}$ Fluor Daniel, South \\ Africa
}

\begin{abstract}
CP 2012 is used extensively throughout the world to design machine foundations for vibrations. The code, however, does not give practical advice on how to design a foundation with piles. CP 2012 models the soil as a system of undamped individual springs. A pile group may similarly be modelled as a system of springs, determined from the geometric and material characteristics of the piles. The stiffness of a pile group is expressed in the same form as sub-grade reactions, permitting the use of the same dynamic equations given in CP 2012; thus, the code may be used for both casessoil and piles. The theory described in this paper is based on structural stiffness theory. Several assumptions are therefore made concerning the soil characteristics, effective length and boundary conditions of the pile. The derivation of the equations are given and compared to a simple finite element model.
\end{abstract}

\section{INTRODUCTION}

Designing foundations with piles for vibrating machinery is a difficult task for the simple reason that practical design methods are not readily available or published in codes of practice (Tomlinson PDCP 1987, British Standards 1974 $[1,2]$. A dearth of information on the subject has caused some designers to resort to commercial computer programmes, such as PIGLET (Randolf PIGLET 2002) [3] and REPUTE (Bond REPUTE 2002) [4], which are capable of analyzing pile groups.

CP 2012, the Code of Practice for Foundations for Machinery (British Standards 1974) [2], is widely used in many parts of the world. The code provides explicit instructions on how to determine natural frequencies and amplitudes of vibration based on the assumption that the foundation can be modeled as a system of undamped individual springs (British Standards 1974, Richart VSF 1970, Irish FRM 1969) [2, 5, 6]. The foundation block is assumed rigid and the spring stiff-nesses are derived from the properties of the soil. The code makes reference to Barkan's (Barkan DBF 1962) [7] method to determine the soil stiffnesses (referred to as subgrade reac-tions). Since the majority of foundations support ing vibrating machinery are founded on soil, the code is applicable to most cases. However, when designing a foundation with piles, the method of design is not explicitly given by the code. There-fore, the objective is to present a simple method to determine the stiffnesses of a pile group which may be used in conjunction with CP 2012.

The proposed method is similar to the design of a foundation block on soil. As mentioned, CP 2012 models the soil as a system of undamped individual springs. A pile group is similarly modeled as a system of springs (Tomlinson PDCP 1987, Poulos GE 1978)[1,8]. The question, however, lies in what approach to determine the stiffnesses of a pile group.

*Address correspondence to this author at the School of Civil and Environmental Engineering, University of the Witwatersrand, South Africa;

E-mail: Mitchell.Gohnert@wits.ac.za
The code suggests either: (i) carry out longitudinal and lateral in situ loading tests on actual piles, or (ii) determining the relevant spring stiffnesses from the geometrical and material configuration of the pile group. Method (i) requires that the piles are already installed prior to the final design. This is the most accurate method, but may prove to be a costly exercise if the piles are found to be inadequate. Method (ii) is therefore the most favourable alternative and is the approach taken by the authors.

The theory described in this paper is based on structural stiffness theory which assumes an effective length and boundary conditions at the end of the pile. However, the effective length and bearing conditions may not be apparent in some soil profiles; in these cases, specialist literature should be referenced.

\section{PILING ASSUMPTIONS}

Sources of resistance to piling forces are shaft friction and end bearing. Vibrations, however, may diminish the frictional resistance along the shaft, particularly in coarse soils. Furthermore, the imposition of lateral forces on the shaft may compact the surrounding soil, resulting in further diminishing the frictional resistance. For these reasons, the frictional resistance along the shaft should be ignored in piles subjected to vibrations. Only where shear and moment fixities of the toe of the pile are considered is stress from the soil on the shaft taken into account. In general, piles applied to vibrating foundations should be "end bearing type" and the shaft assumed to be free.

Longitudinal settlement of the toe of the pile should be negligibly small. To achieve this, the pile should preferably be founded on rock, or sufficiently far into dense or stiff stratum so that an "effective length" of the pile may be estimated. This is the equivalent free length of a pile on a rigid base, such that its longitudinal stiffness is the same as that of the actual pile.

Shear and moment fixities of the toe of the pile are achieved by sufficient embedment of the toe into a stiff and strong stratum. If a pinned toe connection is assumed, only 
shear fixity is required. Although forces developed from shear across the base is usually small, sufficient shear resistance can be developed from penetration of the pile by 1.5 diameters into a stratum classified as "rock".

Moment fixity resistance should only be assumed if powerful drilling equipment has enabled sufficient penetration into a stiff and strong stratum. Specialist geotechnical investigation and assessment of such a situation will be needed.

\section{INITIAL SIZING OF THE FOUNDATION}

Initial sizing of the foundation block is based on empirical rules. The code specifies three basic rules (British Standards 1974) [2].

The first rule requires that the mass of the foundation is greater than the mass of the plant. Although no ratio is provided, usually in practice the mass of the foundation is 3 to 5 times greater than the mass of the plant. This rule of thumb is given to minimize the amplitudes of vibration and is applicable to foundations resting on soil or piles.

The second rule is referred to as the $5 \%$ rule. This requires that the plan geometric centre of the foundation is within $5 \%$ of the centre of mass. Since the dynamic loads are assumed to be applied through the centre of mass, compliance to this rule will ensure an even distribution of stress to soil and therefore an even distribution of settlement. Uneven settlement may result in a further unbalance of forces as well as damage to pipes and equipment. Although this rule is applicable to foundation blocks resting on soil, it is not applicable to foundations supported by piles-however, the notion is the same. An even distribution of force to a set of piles is desirable. Since stiffness attracts force, an even distribution of force is only achieved by applying the force to the centre of stiffness. The 5\% rule can still be applied but stated differently - the centre of mass should be within 5\% of the centre of stiffness, viewed in plan.

The third rule requires that the width of the foundation is at least equal to the distance from centre of the crank shaft of the machine to the bottom of the foundation. This rule is an attempt to ensure stability against overturning. Compliance with the first and the third rule will most likely satisfy stability requirements and is suitable for initial sizing (assuming that the excitation force is not excessive). The final design, however, should include an overturning calculation.

\section{PILE GROUP STIFFNESS EXPRESSED AS A SUB- GRADE REACTION}

The soil stiffness is referred to as the subgrade reaction. The value of stiffness, in units of stress per unit deflection, is called the coefficient of subgrade reaction. Four coefficients are used-the coefficient of uniform compression $\left(c_{u}\right)$, the coefficient of non-uniform compression $\left(c_{\phi}\right)$, the coefficient of uniform shear $\left(c_{r}\right)$ and the coefficient of non-uniform shear $\left(c_{s}\right)$. These coefficients are defined in terms of the pile group stiffnesses, but expressed in the same form as coefficients of subgrade reaction, as described in the following sections 5.1 to 5.4 .

\section{Coefficient of Uniform Compression}

The coefficient of uniform compression is determined from the spring system illustrated in Fig. (1). The foundation block is assumed rigid and the springs schematically represent the elastic properties of the piles.

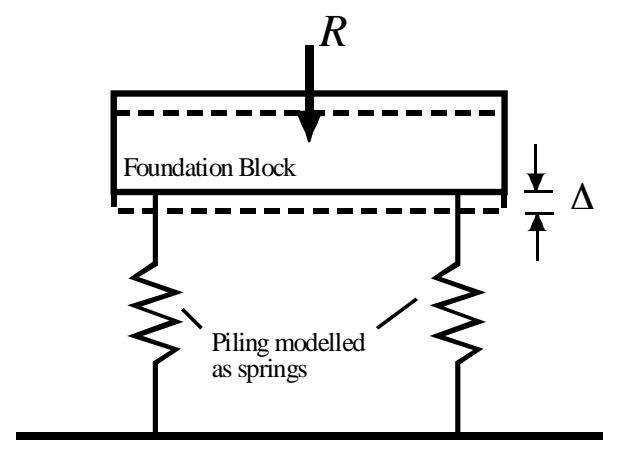

Fig. (1). Foundation spring system for vertical deformations

As a vertical load $(R)$ is applied to the foundation block, a uniform deformation of $\Delta$ will occur in the piles. A pile configuration, where the centre of stiffness coincides with the centre of mass of the machine and foundation, is a necessary prerequisite to ensure a uniform deformation. The vertical stiffness of the piles $\left(k_{v}\right)$, is simply the load divided by the deformation.

$k_{v}=R / \Delta$

Since piles may be vertical or raked, the calculation of stiffness must consider both cases, as illustrated in Fig. (2). As depicted, the total deformation of the pile may be broken down into two fundamental deformations - axial and shear. The axial deformation is represented by the symbol $\Delta_{a}$ and the shear deformation (side sway) is represented by $\Delta_{s}$. Each of these deformations is associated with reactions $R_{a}$ (axial) and $R_{s}$ (shear). The stiffness of the pile depends on the moment fixity of the bearing end.

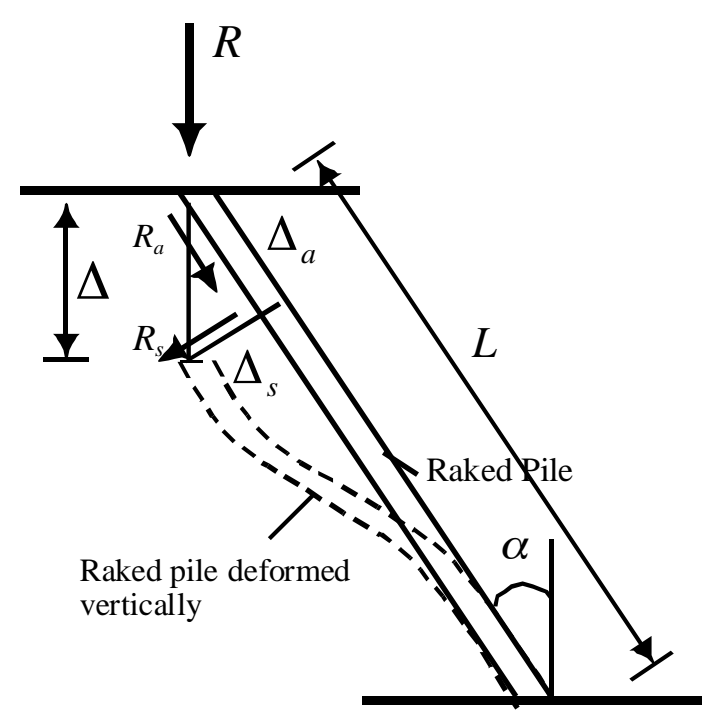

Fig. (2). Vertical deformations of a raked pile. 
Coefficient of Uniform Compression for a Bearing End with Moment Fixity

The stiffness equation for axial deformation is given in equation 2 .

$R_{a}=\left(\frac{E A}{L}\right) \Delta_{a}$

Since,

$\Delta_{a}=\Delta \cos \alpha$

$R_{a}=\left(\frac{E A}{L}\right) \Delta \cos \alpha$

where $E$ is Young's modulus of the pile, $A$ is the crosssectional area of the pile, $L$ is the length of the pile and $\alpha$ is the angle of rake.

Similarly for shear, the stiffness is given by equation 4 .

$R_{s}=\left(\frac{12 E I}{L^{3}}\right) \Delta_{s}$

Since,

$\Delta_{s}=\Delta \sin \alpha$

$R_{s}=\left(\frac{12 E I}{L^{3}}\right) \Delta \sin \alpha$

As shown in Fig. 2, $R_{a}$ and $R_{s}$ are components of $R$.

$R=R_{a} \cos \alpha+R_{s} \sin \alpha$

Substituting equations 3 and 5 ,

$R=\left(\frac{E A}{L}\right) \Delta \cos ^{2} \alpha+\left(\frac{12 E I}{L^{3}}\right) \Delta \sin ^{2} \alpha$

Rearranged,

$R=\left[\left(\frac{E A}{L}\right) \cos ^{2} \alpha+\left(\frac{12 E I}{L^{3}}\right) \sin ^{2} \alpha\right] \Delta=k_{v} \Delta$

The vertical stiffness is the quantity in brackets. The total stiffness is the sum of the stiffnesses of each pile.

$k_{v}=\sum_{1}^{n}\left[\left(\frac{E A}{L}\right) \cos ^{2} \alpha+\left(\frac{12 E I}{L^{3}}\right) \sin ^{2} \alpha\right]$

where $n$ is the number of piles.

The pile group vertical stiffness is expressed in the same form as the subgrade reaction.

$c_{u}=\frac{k_{v}}{A_{p}}$

where $A_{p}$ is the cross-sectional area of the pile group.

Coefficient of Uniform Compression for a Pinned Bearing End

The derivation for the case of a pinned bearing end is similar to the fixed case. The difference is the term of the equation which represents the shear stiffness. The stiffness equation for a pinned bearing end is given in equation 9:

$R=\left[\left(\frac{E A}{L}\right) \cos ^{2} \alpha+\left(\frac{3 E I}{L^{3}}\right) \sin ^{2} \alpha\right] \Delta=k_{v} \Delta$

The stiffness of the pile group is the sum of the stiffnesses of each pile.

$k_{v}=\sum_{1}^{n}\left[\left(\frac{E A}{L}\right) \cos ^{2} \alpha+\left(\frac{3 E I}{L^{3}}\right) \sin ^{2} \alpha\right]$

Eqn 10 is converted into an equivalent subgrade reaction by equation 8 .

\section{Coefficient of Non-Uniform Compression}

The coefficient of non-uniform compression is determined for problems where a non-uniform pressure is applied to the soil. Rocking and pitching moments will cause nonuniform pressures. The foundation block spring system, subjected to a moment, is illustrated in Fig. (3). As before, the springs represent the elastic stiffnesses of the piles.

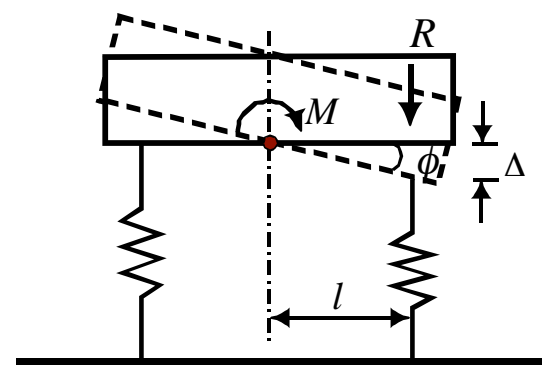

Fig. (3). Foundation spring system for rocking or pitching deformations.

\section{Coefficient of Non-Uniform Compression for Fixed and Pinned Bearing End}

If a rocking or pitching moment $(M)$ is applied, the foundation block will undergo a rotation $(\phi)$. The moment will produce a vertical deflection $\Delta$ and a reaction $R$ in each pile.

The moment contribution of a single pile is given in equation 11 .

$M_{i}=R l$

where $l$ is the distance from the moment axis to the location of the pile.

Since the pile will undergo a vertical deformation, the reactive force is equal to equation 6 or 9 , depending on the end condition of the piles. Substituting equations 6 or 9 into 11 ,

$M_{i}=k_{v} \Delta l$

For small angles of rotation,

$\Delta=\phi l$

$M_{i}=k_{v} l^{2} \phi$

The rotational stiffness is therefore,

$k_{\phi}=k_{v} l^{2}$ 
The total moment $(M)$ is equal to the sum of equation 12 for each pile.

$$
M=\sum_{1}^{n}\left(k_{v} l^{2}\right) \phi
$$

The rotation $\phi$ is a constant and therefore pulled out of the summation.

From Barkan (Barkan DBF1962) [7], the moment is also expressed in terms of the subgrade reaction and the moment of inertia $\left(I_{p}\right)$ of the pile group.

$$
M=c_{\phi} I_{p} \phi
$$

Equating equations 13 and 14, the coefficient of nonuniform compression is solved.

$$
c_{\phi}=\frac{\sum_{1}^{n}\left(k_{v} l^{2}\right)}{I_{p}}
$$

The values $l$ and $I_{p}$ may change in the $\mathrm{x}$ and y directions. For this reason, equations 16 and 17 are defined accordingly.

$$
\begin{aligned}
c_{\phi x}= & \frac{\sum_{1}^{n}\left(k_{v} l_{y}^{2}\right)}{I_{p x}} \\
c_{\phi y} & =\frac{\sum_{1}^{n}\left(k_{v} l_{x}^{2}\right)}{I_{p y}}
\end{aligned}
$$

From equation 14, the rotational stiffness is determined.

$$
k_{\phi}=c_{\phi} I_{p}
$$

Substituting equations 16 and 17 into 18 , the $\mathrm{x}$ and $\mathrm{y}$ stiffnesses for non-uniform compression is solved.

$$
\begin{aligned}
& k_{\phi x}=\sum_{1}^{n}\left(k_{v} l_{y}^{2}\right) \\
& k_{\phi y}=\sum_{1}^{n}\left(k_{v} l_{x}^{2}\right)
\end{aligned}
$$

\section{Coefficient of Uniform Shear}

The coefficient of uniform shear is derived from the interaction of the horizontal forces on the soil. The foundation block spring system is illustrated in Fig. (4). As shown, the springs are placed horizontally to represent the shear stiffness of the soil. A raked pile is assumed to be deformed in the horizontal direction as shown in Fig. (5). Similar to the case of uniform compression, the total deformation is broken down into two fundamental deformations-axial and shear (side sway). The axial deformation is represented by the symbol $\Delta_{a}$ and the shear deformation is represented by $\Delta_{s}$. These deformations are associated with reactions $R_{a}$ (axial) and $R_{s}$ (shear).

\section{Coefficient of Uniform Shear for Fixed Bearing End}

The stiffness equation for axial deformation is given by equation 21 .

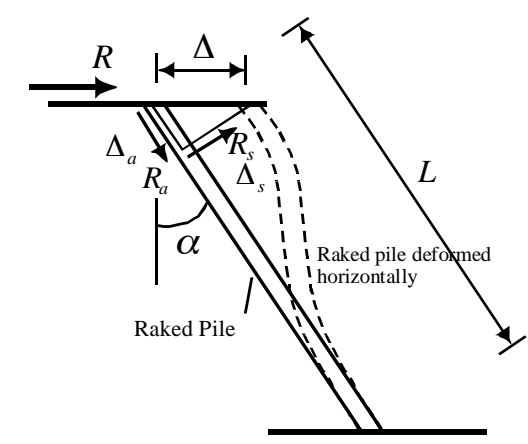

Fig. (4). Foundation spring system for horizontal deformations.

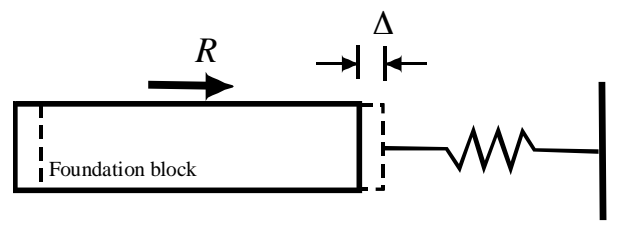

Fig. (5). Horizontal deformations of a raked pile.

$R_{a}=\left(\frac{A E}{L}\right) \Delta_{a}$

Since,

$\Delta_{a}=\Delta \sin \alpha$

$R_{a}=\left(\frac{A E}{L}\right) \Delta \sin \alpha$

The stiffness for the shear (side sway) is given by equation 23 .

$R_{s}=\left(\frac{12 E I}{L^{3}}\right) \Delta_{s}$

Since,

$\Delta_{s}=\Delta \cos \alpha$

$R_{s}=\left(\frac{12 E I}{L^{3}}\right) \Delta \cos \alpha$

The reactions $R_{a}$ and $R_{s}$ are components of $R$.

$R=R_{a} \sin \alpha+R_{s} \cos \alpha$

Substituting equations 22 and 24,

$R=\left(\frac{A E}{L}\right) \Delta \sin ^{2} \alpha+\left(\frac{12 E I}{L^{3}}\right) \Delta \cos ^{2} \alpha$

Rearranged,

$R=\left[\left(\frac{A E}{L}\right) \sin ^{2} \alpha+\left(\frac{12 E I}{L^{3}}\right) \cos ^{2} \alpha\right] \Delta=k_{h} \Delta$

The horizontal stiffness $\left(k_{h}\right)$ is the quantity in brackets. The total stiffness is the sum of the stiffnesses of each pile.

$k_{h}=\sum_{1}^{n}\left[\left(\frac{A E}{L}\right) \sin ^{2} \alpha+\left(\frac{12 E I}{L^{3}}\right) \cos ^{2} \alpha\right]$ 
The horizontal stiffness of the pile group is expressed in the same form as the subgrade reaction.

$c_{r}=\frac{k_{h}}{A_{p}}$

If the piles are raked, the horizontal shear stiffness may differ in the $\mathrm{x}$ and $\mathrm{y}$ directions, depending on the direction of rake. For this reason, the horizontal stiffnesses are determined in the $\mathrm{x}$ and $\mathrm{y}$ directions $\left(k_{h x}\right.$ and $\left.k_{h y}\right)$.

In the direction of rake, the pile stiffness is based on the axial and side sway deformations of the pile. If the pile is not raked, the stiffness equation will only include side sway. To account for this, the rake angle $\alpha$ is replaced by $\alpha \cos ^{2} \beta$ to determine the horizontal stiffness in the $\mathrm{x}$ direction and $\alpha$ is replaced by $\alpha \sin ^{2} \beta$ to determine the horizontal stiffness in the y direction. The angle $\beta$ is the angle in plan of a raked pile, as shown in Fig. (6). The horizontal stiffnesses in the $\mathrm{x}$ and y directions are given in equations 29 and 30.

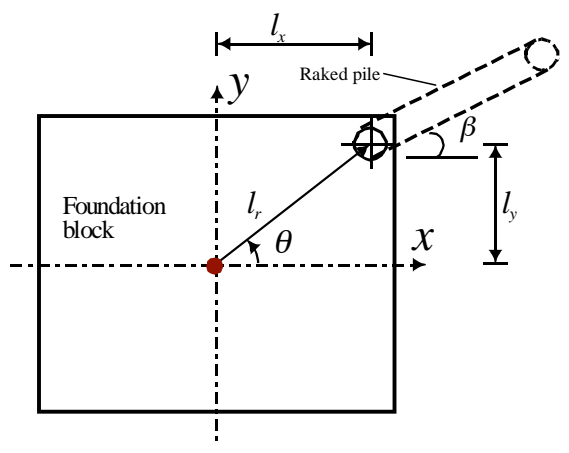

Fig. (6). Angle orientation of raked piles in plan.

$$
\begin{aligned}
& k_{h x}=\sum_{1}^{n}\left[\left(\frac{A E}{L}\right) \sin ^{2}\left(\alpha \cos ^{2} \beta\right)+\left(\frac{12 E I}{L^{3}}\right) \cos ^{2}\left(\alpha \cos ^{2} \beta\right)\right] \\
& k_{h y}=\sum_{1}^{n}\left[\left(\frac{A E}{L}\right) \sin ^{2}\left(\alpha \sin ^{2} \beta\right)+\left(\frac{12 E I}{L^{3}}\right) \cos ^{2}\left(\alpha \sin ^{2} \beta\right)\right]
\end{aligned}
$$

Furthermore, the coefficients of subgrade reactions are defined in the $\mathrm{x}$ and $\mathrm{y}$ directions.

$$
\begin{gathered}
c_{r x}=\frac{k_{h x}}{A_{p}} \\
c_{r y}=\frac{k_{h y}}{A_{p}}
\end{gathered}
$$

\section{Coefficient of Uniform Shear for Pinned Bearing End}

The derivation of the coefficient of uniform shear for a pinned bearing end is similar to the derivation of the fixed bearing end case. The difference is the horizontal shear stiffness term.

$$
\begin{aligned}
& k_{h x}=\sum_{1}^{n}\left[\left(\frac{A E}{L}\right) \sin ^{2}\left(\alpha \cos ^{2} \beta\right)+\left(\frac{3 E I}{L^{3}}\right) \cos ^{2}\left(\alpha \cos ^{2} \beta\right)\right] \\
& k_{h y}=\sum_{1}^{n}\left[\left(\frac{A E}{L}\right) \sin ^{2}\left(\alpha \sin ^{2} \beta\right)+\left(\frac{3 E I}{L^{3}}\right) \cos ^{2}\left(\alpha \sin ^{2} \beta\right)\right]
\end{aligned}
$$

The equivalent coefficient of subgrade reaction is determined by substituting equations 33 and 34 into equations 31 and 32.

\section{Coefficient of Non-Uniform Shear for Fixed and Pinned Bearing End}

The coefficient of non-uniform shear represents the stiffness of the soil determined from the twisting (or drilling or yawing) action of the foundation base. The applied moment is referred to as the yawing moment. The foundation block spring system is illustrated in Fig. (7). From the figure, the radius $\left(l_{r}\right)$ is the distance from the axis of the yawing moment to the location of the pile. The direction of deformation $(\Delta)$ and the calculated stiffness is orientated at right angles to the radius. The deformation is a function of the shear reaction $\left(R_{s}\right)$ and the angle of twist $(\psi)$.

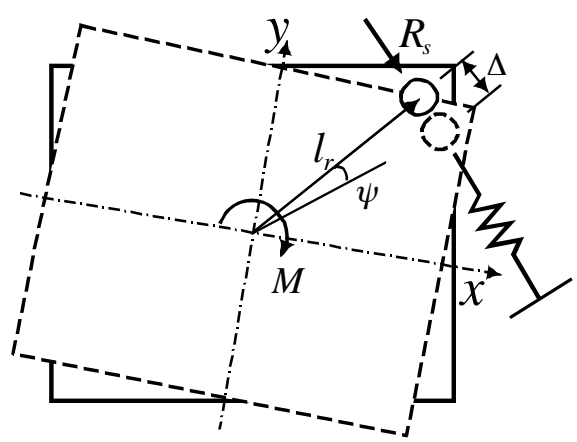

Fig. (7). Foundation spring system of twisting deformations.

The moment contribution of a single pile is equal to the horizontal shear reaction times the radial moment arm $\left(l_{r}\right)$.

$M_{i}=R_{s} l_{r}$

The horizontal reaction is equal to equation 26 , at right angles to the radius.

$M_{i}=k_{h \theta} \Delta l_{r}$

Where $k_{h \theta}$ is the horizontal stiffness at an angle $\theta$ (at right angles to the radius).

For a small angle of twist,

$$
\begin{aligned}
& \Delta=l_{r} \psi \\
& M_{i}=l_{r}^{2} k_{h \theta} \psi
\end{aligned}
$$

The total yawing moment is equal to the sum of $M_{i}$ for each pile.

$$
M=\sum_{1}^{n}\left(l^{2} k_{h \theta}\right) \psi
$$

From Barkan ${ }^{7}$, the twisting moment is related to the polar moment of inertia $(J)$ and the subgrade reaction $c_{s}$.

$$
M=c_{s} J \psi
$$

Where $J$ is the polar moment of inertia of the pile group.

Equating equations 36 and 37, the coefficient of nonuniform shear is determined. 


$$
c_{s}=\frac{\sum_{1}^{n}\left(l_{r}{ }^{2} k_{h \theta}\right)}{J}
$$

From equation 37, the twisting stiffness is determined.

$k_{\psi}=c_{s} J$

Combining equations 38 and 39 ,

$$
k_{\psi}=\sum_{1}^{n}\left(l_{r}^{2} k_{h \theta}\right)
$$

The horizontal stiffness $\left(k_{h \theta}\right)$ is the stiffness at right angles to the radius $\left(l_{r}\right)$.

$$
k_{h \theta}=k_{h x} \sin ^{2} \theta+k_{h y} \cos ^{2} \theta
$$

\section{VALIDATION OF STIFFNESS EQUATIONS}

A simple pile configuration, supporting a vibrating foundation, is illustrated in Fig. (8). The four piles are raked (1:5), the effective length 2.65 metres long ( $2.7 \mathrm{~m}$ along the rake) and assumed fixed at both ends. The pile is end bearing, cast into an underlying rock stratum and assumed fully fixed at the pile tip. The piles are raked in one direction along the $\mathrm{x}$-axis. Other parameters, defining the properties of the piles are given below:

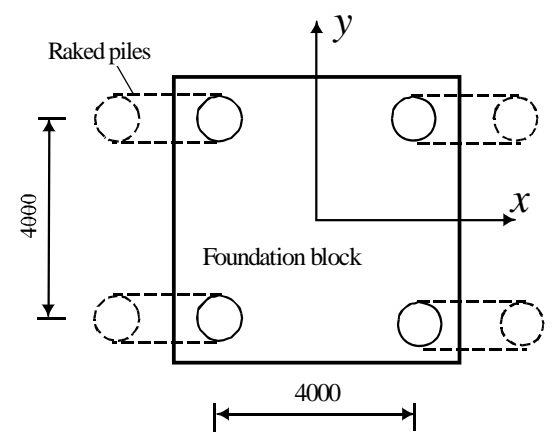

Fig. (8). Pile configuration of a foundation block.

Pile diameter $=600 \mathrm{~mm}$

$$
f_{c u}=30 \mathrm{MPa}
$$

$\mathrm{E}($ dynamic $)=38 \mathrm{GPa}$

$\mathrm{I}=6.36 \times 10^{-3} \mathrm{~m}^{4}$ (single pile)

$\mathrm{I}_{\mathrm{px}}=\mathrm{I}_{\mathrm{py}}=4.55 \mathrm{~m}^{4}$ (pile group)

$\mathrm{A}=2.83 \times 10^{-1} \mathrm{~m}^{2}$ (single pile)

$\mathrm{A}_{\mathrm{p}}=1.13 \mathrm{~m}^{2}$ (pile group)

$\mathrm{J}=1.27 \times 10^{-2} \mathrm{~m}^{3}$ (pile group)

The proposed stiffness equations are compared to an ABAQUS finite element solution. The model uses beam elements to represent the piles and shell elements (with an exaggerated $E$ to make the block rigid) to model the block foundation. The stiffnesses of the pile group are determined by applying arbitrary forces to the finite element configuration and solving for the deformations (or rotations). The stiffnesses are calculated by dividing the force by the deformation or by dividing the moment by the rotation. The re- sults are compared with the proposed theory and compiled in Table 1. Since the derivation of the theory is based on "first principles," the equations are nearly identical to the finite element model.

Table 1. Comparison of Stiffness Equations

\begin{tabular}{|c|c|c|}
\hline $\begin{array}{c}\text { Pile Stiffness } \\
\text { (4 Pile Grouping) }\end{array}$ & $\begin{array}{c}\text { Stiffness Equations } \\
\text { (i.e., Eqns 7, 16, 17, 26, } \\
\mathbf{2 7} \text { and 36) } \\
\text { (N/m) }\end{array}$ & $\begin{array}{c}\text { Finite Element } \\
\text { Solution } \\
\text { (N/m) }\end{array}$ \\
\hline \hline$k_{v}$ & $1.53 \times 10^{10}$ & $1.54 \times 10^{10}$ \\
\hline$k_{\phi x}$ & $6.12 \times 10^{10}$ & $6.32 \times 10^{10}$ \\
\hline$k_{\phi y}$ & $6.12 \times 10^{10}$ & $6.32 \times 10^{10}$ \\
\hline$k_{h x}$ & $1.18 \times 10^{9}$ & $1.18 \times 10^{9}$ \\
\hline$k_{h y}$ & $5.89 \times 10^{8}$ & $5.88 \times 10^{8}$ \\
\hline$k_{\psi}$ & $7.07 \times 10^{9}$ & $7.05 \times 10^{12}$ \\
\hline
\end{tabular}

\section{EFFECTIVE LENGTH OF THE PILES}

In practice, it is often difficult to determine the effective length of a pile, due to a variation in soil properties along the profile. The question is whether the effective length extends to the depth of refusal of boring or driving, or if the soil is sufficiently stiff to provide fixity at a higher level? The example given above is taken from an actual design. The soil profile consisted of a silty sand hillwash and sandy clay residual dolerites to a depth of $2.2 \mathrm{~m}$. From $2.2 \mathrm{~m}$ to $5.5 \mathrm{~m}$, the profile consisted of a soft rock dolerite. Although refusal occurred at $5.5 \mathrm{~m}$, the effective length was assumed to be equal to the extent of the sandy clay residual dolerites $(2.2$ m) plus 0.75 times the diameter of the pile-the soft rock was assumed to be sufficiently rigid to provide fixity. This assumption was substantiated by a load/settlement test on one of the piles. Load/settlement tests are common and specified by codes of practice (South African Bureau of Standards 1983) [9]. The data for the single pile test is given in Fig. (9). The pile was initially loaded to $100 \%$ of working load to allow the pile to "settle in." The load was removed and reapplied to full working load. The effective length of the pile was estimated by an elastic axial load/deflection relationship.

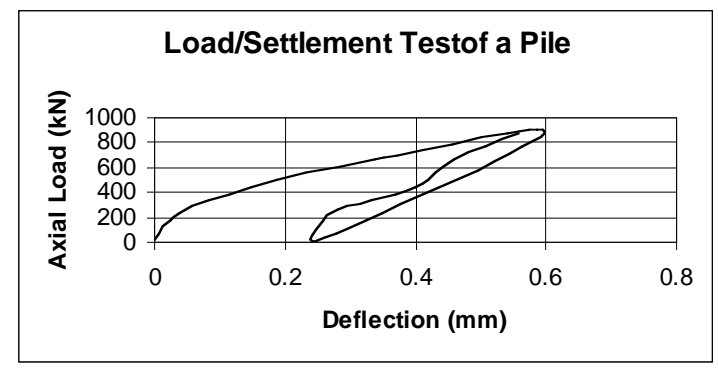

Fig. (9). Results of a load/settlement test on a pile.

$l_{\text {eff }}=\frac{\delta A E}{R}$ 
where $\delta$ is the axial deflection, $A$ is area of the pile, $E$ is the elastic modulus and $R$ is the axial load.

The effective length was calculated at $2.82 \mathrm{~m}$, which is reasonably close to the initial estimate of $2.65 \mathrm{~m}$. It should be noted that the deflection $(\delta)$ is the recorded deflection at $100 \%$ working load less the residual deflection once the load is removed at the end of the first cycle of loading. Since the loading was applied over a period of 24 hours, the elastic modulus is based on static loads. Although the usual objective of load testing is to determine the in-situ settlement of a pile, the data is useful to estimate the effective length.

Although the above provides a simple and convenient method of estimating the effective length, the actual effective length is the result of a host of complex interactions between the pile and the surrounding soil. Initially and possibly during the life of the foundation, the vertical and lateral stiffness of the soil may be larger than the stiffness of the piles. However, under dynamic conditions, the influence of the surrounding soil is greatest at the beginning stages of vibration loading, but wanes with time as the soil is compacted and so leaves a gap around the shaft. Therefore the above method is flawed in the sense that the test is static and the real load is dynamic. All of these factors should be considered when estimating the effective length and specialist literature should be consulted for a more exact estimate (Cairo CG 2005) [10].

An in-situ test, however, potentially provides a close approximation of the effective length and boundary condition at the toe, particularly if axial strain measurements along the shaft in the test enable force distribution and hence surface shear stress v. shaft displacement relationships along the shaft to be determined. With these relationships, the "load transfer" method of determining the stiffness of the pile can be used (Coyle and Reese 1966) in which the contribution of upper soil strata where compaction may occur can be ignored.

\section{CONCLUSIONS}

The proposed equations are a simplified method to model piling in a form that is adaptable to CP 2012. The theory, however, dictates that the pile must be end bearing, unrestrained along the shaft and fixed or pinned at the bearing end. Although many pile types rely on skin friction, piles subjected to vibrations potentially lose the frictional bond between the soil and the shaft. For this reason, an unrestrained shaft is assumed (Tomlinson PDCP 1987) [1].

When applying the vibration equations of CP 2012, one should bear in mind that the values of $A, I$ and $J$ are values determined for a pile group, and are not associated with the base of the foundation. The analysis, however, does not include that which is commonly referred to as the "group effect" in the analysis of pile groups. This essentially results in the resistance of a group of piles being less than the resis- tance of the same number of piles acting in isolation. For example Poulos and Randolph [11]. However, if degradation of the interaction between the pile shaft and soil by vibratory loads does take place, then this group effect will be small, and the conservative approach recommended here, to ignore the shaft to soil interaction except near the toe where in very strong strata it can provide fixity, is expected to offset the group effect [12].

Table 1 indicates that the proposed equations (which are derived from first principles) compare well with an ABAQUS finite element solution and therefore gives credence to the theory. Furthermore, since the properties of concrete are more predictable than soils, stiffnesses determined from a pile group are potentially more accurate than the stiffnesses determined from soil samples. Thus, a vibration analysis of a block foundation on a pile group is potentially more accurate than a foundation on soil.

As illustrated in the example problem, vertical load tests on piles can be used to estimate the effective length. This is particularly useful when the soil profile is such that the effective length cannot be clearly defined by inspection. However, this method of determining the effective length is applicable to foundations that are subjected primarily to vertical vibrations. Where other modes of vibration are dominant, other testing methods or specialty literature should be sought.

\section{REFERENCES}

[1] M.J. Tomlinson, Pile Design and Construction Practice ( $3^{\text {rd }}$ ed), London: Viewpoint, 1987.

[2] British Standards Institute, CP 2012: Part 1 Code of Practice for Foundations for Machinery, BSI, London, 1974.

[3] M.F. Randolf, PIGLET: Analysis and Design of Pile Groups, Users' Manual, Version 4-2, University of Western Australia, Australia, 2002.

[4] A.J. Bond and F. Basile, REPUTE 1.0, Pile Group Design Software, Users' Manual, Geocentrix Ltd., London, 2002.

[5] F.E. Richart, R.D. Woods and J.R. Hall, Vibrations of Soils and Foundations, New Jersey: Prentice-Hall, 1970.

[6] K. Irish and W.P. Walker, Foundations for Reciprocating Machines, London: Concrete Publications, 1969.

[7] D.D. Barkan, Dynamics of Bases and Foundations, New York: McGraw-Hill, 1962.

[8] H.G. Poulos and E.H. Davis, Pile Foundation Analysis and Design, Series in Geotechnical Engineering, T.W. Lambe, and R.V. Whitman, Eds. Massachusetts Institute of Technology, John Wiley \& Sons, Massachusetts, 1978.

[9] South African Bureau of Standards, SABS 1200 F Piling, Pretoria, 1983.

[10] R. Cairo, E. Conte and G. Dente, "Analysis of pile groups under vertical harmonic vibration", Computers and Geotechnics, Vol. 32(7), pp. 545-54, 2005.

[11] H.G. Poulos and M.F. Randolph, "Pile group analysis: a study of two methods", Journal of Geotechnical Engineering, ASCE, Vol. 109, No. 3, pp. 355-372, March 1983.

[12] H.M. Coyle and L.C. Reese, "Load transfer for axially loaded piles in clay". Journal of Soil Mechanism and Foundation Engineering Division, ASCE 92, SM2, pp. 1B126, 1966. 